\title{
Precise Peptide Sequencing and Protein Quantification in the Human Proteome Through In Vivo Lysine-Specific Mass Tagging
}

\author{
Sheng Gu, ${ }^{\dagger}$ Songqin Pan, ${ }^{\dagger}$ E. Morton Bradbury, ${ }^{\ddagger}$ and Xian Chen
}

Bioscience Division, Los Alamos National Laboratory, Los Alamos, New Mexico, USA

\begin{abstract}
Proteomics studies demand new scalable and automatable MS-based methods with higher specificity and accuracy. Here we describe an accurate and efficient method for both precise quantification and comprehensive de novo identification of peptide sequences in complex mixtures. The unique feature of this method is based on the incorporation of deuteriumlabeled (heavy) lysines into proteins through in vivo cell culturing, which introduces specific mass tags at the carboxyl termini of proteolytic peptides when cleaved by certain proteases. The mass shift between the unlabeled and the deuterated lysine (lys- $\left.d_{4}\right)$ assigns a mass signature to all lysine-containing peptides in any pool of proteolytic peptides. Lys- $d_{4}$ tags can also serve as internal markers in MS/MS fragment spectra when they are buried in some peptide sequences due to miscleavages. This signal specificity circumvents the mass accuracy limitations in determining particular amino acid residues for de novo sequencing. Further, this strategy of lysine-specific tagging was successfully implemented to measure the differential protein expression of human skin fibroblast cells in response to heat shock. (J Am Soc Mass Spectrom 2003, 14, 1-7) () 2003 American Society for Mass Spectrometry
\end{abstract}

$\mathrm{W}$ e present here a highly specific and efficient in vivo method for de novo peptide sequencing and protein quantification that is complimentary to in vitro methods such as isotope-coded affinity tags (ICAT) [1] and mass-coded abundance tagging (MCAT) [2]. Protein identification through peptide de novo sequencing is essential for proteomic studies of complex organisms [3-6]. Current methods for peptide de novo sequencing rely largely on chemical modifications of certain amino acid residues that provide specific mass tags for deriving peptide sequences de novo [5-11]. However, these are time-consuming multi-step approaches that are amenable to only a few amino acids. For example, cysteine is present at low abundance in the human proteome, and additionally, post-translational modifications of cysteines make them less reactive to the ICAT reagent [7]. The MCAT approach cannot identify internal lysine(s) in peptides that result from frequent miscleavages in tryptic digests. In this report, the incorporation of deuterium-labeled

Published online November 28, 2002

Address reprint requests to Dr. X. Chen, MS M888, Bioscience Division, Los Alamos National Laboratory, Los Alamos, NM 87545, USA. E-mail: chen_xian@lanl.gov

$\ddagger$ Also at the Department of Biological Chemistry, School of Medicine, University of California at Davis, Davis, CA 95616.

tAuthors contributed equally to this work. (heavy) lysines (lys- $d_{4}$ ) into proteins through in vivo cell culturing eliminates the chemical processes required for chemical tagging. Because lysine is an essential amino acid to human cells, there is no metabolic pathway in lysine synthesis, and thereby, no isotope scrambling with lys- $d_{4}$ precursors should be expected. These heavy lysine-labeled proteins maintain the same ionization and CID efficiency as unlabeled proteins. They provide an intrinsic reference for quantification, and by the accurate assignment of the different series of fragment ions simplify the interpretation of the MS/MS spectra, greatly improving the specificity and accuracy of peptide sequencing and quantification.

\section{Experimental}

Mass Tagging HSF Cells with the lys- $d_{4}$ Precursors and Heat Shock Procedure

The L-4,4,5,5- $\mathrm{D}_{4}$-lysine.2HCl precursor (lys- $d_{4}$ ) was purchased from Cambridge Isotope Laboratories (Andover, MA). All chemicals for culturing including the $\alpha$-MEM medium (formula 32561), the dialyzed fetal bovine serum, and antibiotics were obtained from GIBCO-BRL (Grand Island, NY). The $\alpha$-MEM medium is made of 20 amino acids plus cystine, inorganic salts, trace elements, etc. The concentration of each component is as indicated 
in the manufacturer's manual. The human skin fibroblast (HSF) cells were grown in the $\alpha$-MEM medium at $37{ }^{\circ} \mathrm{C}$ with $5 \% \mathrm{CO}_{2}$ and $90 \%$ relative humidity [12]. The medium was supplemented with $10 \%$ dialyzed fetal bovine serum, $100 \mathrm{u} / \mathrm{ml}$ penicillin and $100 \mu \mathrm{g} / \mathrm{ml}$ streptomycin sulfate. To achieve $50 \%$ lys- $d_{4}$ labeling for observing 4-Da mass splits, $44 \mathrm{mg}$ lys- $d_{4}$ and $36.5 \mathrm{mg}$ natural occurring or unlabeled L-lysine were added to 1 liter lysine-depleted medium which originally contains no lysine to give an equal molar concentration of both labeled and unlabeled lysine in the medium. For the $100 \%$ lys- $d_{4}$ labeling, $88 \mathrm{mg}$ per liter of lys- $d_{4}$ precursors was added to the lysine-depleted $\alpha$-MEM medium. At about $80 \%$ confluent, cells were harvested with trypsin-EDTA treatment. For heat shock treatment, the culturing flasks were removed from the $37^{\circ} \mathrm{C}$ incubator and the medium was changed with fresh medium pre-incubated at $43{ }^{\circ} \mathrm{C}$. Then, the flasks were placed in $43^{\circ} \mathrm{C}$ incubator for $8 \mathrm{~h}$. After the cells were harvested, the same numbers of the unlabeled, non heat-treated cells and the $100 \%$ lys- $d_{4}$-labeled, heattreated cells were mixed. The cells were washed with phosphate buffer saline (PBS buffer) and then lysed with SDS loading buffer, and the total lysate supernatants of approximately 3 million cells were subject to 1-D SDS PAGE separation. The protein bands of interest were excised for MS analysis. In-gel digestion with trypsin and peptide extraction was carried out as previously described [13].

\section{Liquid Chromatography-Electrospray Ionization Mass Spectrometry}

A $\mu$-flow HPLC system (Beckman Coulter, Fullerton, CA) configured with a $1.0 \times 100 \mathrm{~mm}$ Aquasil C18 (Keystone, Bellefonte, PA) was used in LC-MS experiments coupled with a LCQ Deca ion trap mass spectrometer (Thermofinnigan, San Jose, CA). The LC flow rate was set at $50 \mu \mathrm{l} / \mathrm{min}$ and all the eluate went into the MS without splitting the flow. Mobile phase A was $0.1 \%$ TFA in water and mobile phase B was $0.1 \%$ TFA in acetonitrile. The gradient was kept $2 \%$ B for 2 min, then linear from 2 to $65 \%$ B in $20 \mathrm{~min}, 65 \%$ to $85 \%$ B in $3 \mathrm{~min}$, and kept $85 \%$ B for $3 \mathrm{~min}$, followed by 85 to $2 \%$ B in 3 min. The ion trap mass spectrometer was operated at Ispray voltage $3.5 \mathrm{kV}$, and the other parameters were tuned using $500 \mathrm{fmol} / \mu \mathrm{l}$ human Angiotensin I solution to ensure the optimal working condition. The ion trap was operating in data dependent scan mode, and the parent ion isolation width is $2.0 \mathrm{Da}$. A similar approach was also carried out on Micromass Q-TOF Ultima equipped with a capillary LC system (Micromass, Beverly, MA) interfaced by a nanospray source to improve the sensitivity and the mass accuracy. The RP-HPLC separation was carried out on a $150 \mathrm{~mm}$ long $75 \mu \mathrm{m}$ i.d. PepMap $\mathrm{C}_{18}$ microcapillary column (LC Packings, San Francisco, CA) at a flow rate of $250 \mathrm{nl} / \mathrm{min}$. Mobile phase $\mathrm{A}$ was $3 \%$ acetonitrile in $\mathrm{H}_{2} \mathrm{O}$ with $0.1 \%$ formic acid and mobile phase $\mathrm{B}$ was $3 \% \mathrm{H}_{2} \mathrm{O}$ in acetonitrile with $0.1 \%$ formic acid. The gradient was kept $5 \%$ B for $3 \mathrm{~min}$, then ramped linearly to $50 \% \mathrm{~B}$ in $30 \mathrm{~min}$. The parent ion isolation width in Q-TOF was 5.0 Da to isolate both lys- $\mathrm{d}_{4}$-labeled and unlabeled peak for fragmentation simultaneously.

\section{MALDI-TOF Mass Spectrometry}

All MALDI-TOF MS experiments were carried out on a PE Voyager DE_STR biospectrometry workstation (PE Biosystem, Framingham, MA) equipped with a $\mathrm{N}_{2}$ laser (337 nm, $3 \mathrm{~ns}$ pulse width, $20 \mathrm{~Hz}$ repetition rate) in reflector modes. The $m / z$ values were calibrated by peptide standard mixture (angiotensin II, ACTH 1-17 and Insulin Chain B Oxide, PE Biosystem).

\section{Database Search}

The lys- $d_{4}$-assisted de novo sequencing results were confirmed by submitting the same sets of data to the SEQUEST or BioLynx program for protein identification using the Human protein database from NCBI. The search results were verified by the mass-split pattern of the lys- $d_{4}$ labeling. Peptide mass mapping (PMM) data obtained from MALDI-TOF MS analysis was submitted to MS-Fit (http:/ / prospector.ucsf.edu) to search against the Homo Sapiens species in NCBI database.

\section{Results and Discussion}

HSF cells were grown in control medium and medium containing lys- $d_{4}$ precursor. Cells were lysed and the lysate separated by 1-D SDS PAGE. Bands selected for MS analysis were digested in-gel with trypsin to obtain their peptide mass maps (PMM) through MALDI-TOF or ESI MS. Figure 1 shows a 1-D gel image of the supernatant of the HSF cell lysate and the MALDI TOF mass spectrum of a $1-\mathrm{D}$ protein band from the $50 \%$ lys- $d_{4}$ labeled HSF cell lysate. There are several peaks showing the 4 Da mass-split patterns while other peptide signals displayed no split in the MALDI TOF MS PMM. After data searching for the PMM, all those mass-split peaks were found to be lysine-containing peptides. Moreover, no isotopic scrambling was observed in MALDI TOF spectra of all 1-D protein bands containing lys- $d_{4}$ labels. Therefore, in vivo incorporating lys- $d_{4}$ precursors into the HSF proteome has been proven to be highly specific. Meanwhile, other isotopeenriched amino acid precursors can also be selected for in vivo mass tagging of human proteome (Pan et al., manuscript submitted). Considering the high sensitivity of MS-based approaches, the material from a small scale cell culturing will be sufficient for MS analysis and the cost of the stable isotope-labeled precursors will be minimum.

Because lysine is one of the cleavage sites of trypsin, in vivo mass tagging proteins with lys- $d_{4}$ can introduce a mass signature at C-termini of some tryptic peptides, 


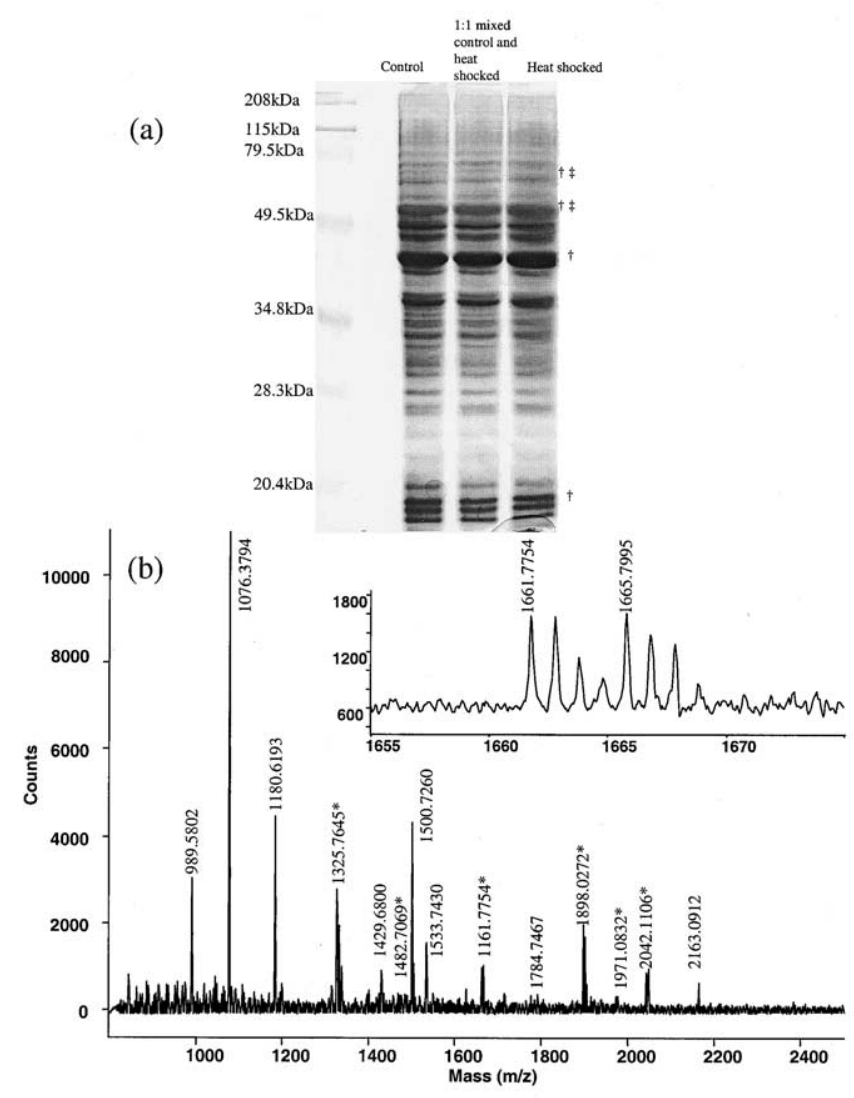

Figure 1. (a) A 1-D SDS PAGE gel image of the HSF cell lysate; single dagger indicates the protein bands studied, double dagger refers protein bands containing the heat-shock induced proteins. (b) MALDI-TOF mass spectrum of an in-gel tryptic digestion of a protein band labeled with $50 \%$ lys- $d_{4}$ isolated from 1-D gel. The peptide signals showing the characteristic $4 \mathrm{Da}$ mass splits are marked with an asterisk. The inset shows an expanded view of the peptide at $m / z 1661.7754$. All these peptides were confirmed as coming from lysine-containing peptides and no isotope scrambling was observed in the PMM.

which can be used to assist de novo peptide sequencing. Those peptides showing characteristic 4-Da mass-splits were subject to MS/MS fragmentation (Figure 2a, b, and c). To illustrate how lys- $d_{4}$ tags help to distinguish different fragment ions, Figure $2 \mathrm{~b}$ and $\mathrm{c}$ show the MS/MS spectra for the unlabeled (Figure $2 b$ ) and lys- $d_{4}$ labeled (Figure 2c) species. A 4-Da mass shift with respect to their unlabeled counterparts was observed for each fragment ion that originated from the lys- $d_{4^{-}}$ labeled parent peptide, indicating the presence of lysine in the $y$-series ions. Because daughter fragments in MS/MS spectra are predominately in single- or doublecharged states, the 4- or 2-Da mass difference, respectively, is fully resolvable in any commercial MS instrument. In contrast, the $b$-series of ions from the $\mathrm{N}$-termini of peptides from either unlabeled or lys- $d_{4}$-labeled cells remain identical in mass because of the absence of lysine in these fragments. Therefore, the characteristic mass-split pattern for all lysine-containing ions is readily distinguishable from other series of fragments. After the identification of the $y$-series lysine-containing ions in Figure 2c, the identity of neighboring amino acid residue(s) in this peptide was easily determined by the mass difference between two adjacent $y$ ions. Further, because the $\mathrm{N}$-terminal or $b$-series ions were also unambiguously identified through the spectral filtration of lys- $d_{4}$-specific product ions, peptide sequences could also be deduced from the complimentary $b$-series ions for the regions missing $y$-series ions.

Significantly, the lys- $d_{4}$ signature can resolve ambiguities resulting from incomplete tryptic digestion that occurs frequently in complex protein mixtures or from the mass degeneracy between lysine (128.0950 Da) and glutamine (128.0586 Da). For example, lys- $d_{4}$-assisted de novo sequencing was performed on some Q-TOFproduced MS/MS spectra that were interpreted incorrectly using both SEQUEST and Biolynx (Micromass) data search programs despite the ultrahigh mass accuracy of Q-TOF MS (30 mDa). The Q-TOF MS/MS spectrum (Figure 3a) displays both the unlabeled and lys- $d_{4}$-labeled forms of individual daughter fragments derived from a tryptic peptide from the $50 \%$ lys- $d_{4}$ labeled HSF cells. This intact peptide at $1586.79 \mathrm{Da}$ showed the characteristic mass-split patterns in both $2+$ and $3+$ charge states and was selected for CID fragmentation. Interestingly, unlike the typical MS/MS 
(a)

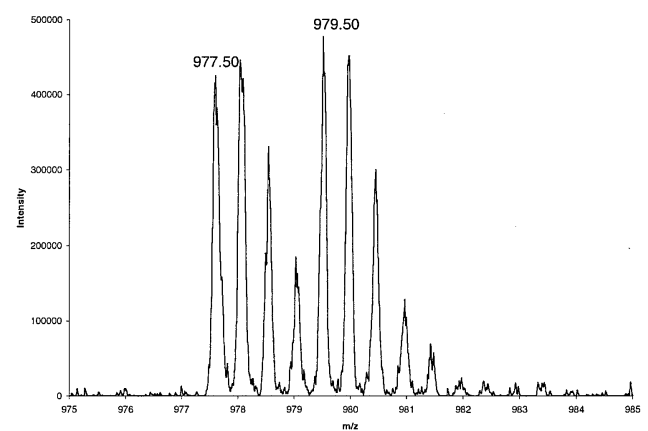

(b)

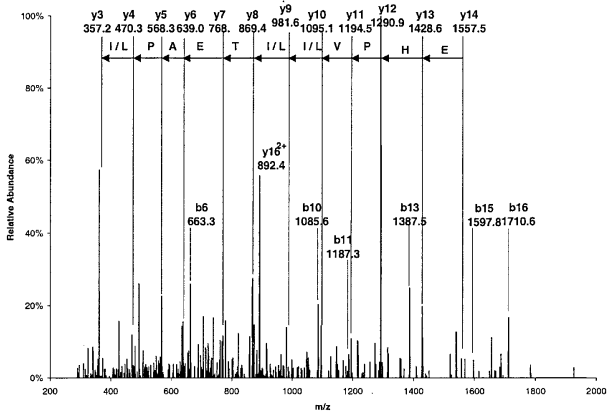

(c)

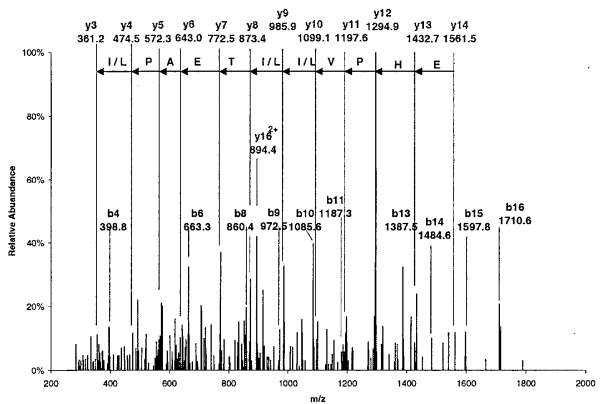

Figure 2. Electrospray mass spectra of the $50 \%$ lys- $d_{4}$ labeled peptide (VAPEEHPVLLTEAPLNPK) with molecular weight 1953.0 Da from an in-gel tryptic digestion of a 1-D SDS PAGE gel band at $45 \mathrm{kDa}$. (a) Zoom scan spectrum from the range of $\mathrm{m} / \mathrm{z} 975$ to 985 . The monoisotopic peak of the unlabeled peptide is at 977.50 , whereas the lys- $d_{4}$ labeled signal is at 979.50 , i.e., 2 Da mass split. The mass difference between adjacent isotopic peaks was 0.5 $\mathrm{Da}$, indicating that these parent ions were doubly charged. (b) MS/MS spectrum of the unlabeled peptide. (c) MS/MS spectrum of lys- $d_{4}$ labeled peptide. There was a $4 \mathrm{Da}$ shift in the y series of ions resulting from the lys- $d_{4}$ labeling.

fragment patterns for other peptides all containing lysine only at the C-terminus, the mass-split pattern, i.e., $4 \mathrm{Da}$ for a single-charged or $2 \mathrm{Da}$ for a doublecharged ion (Figure 3b), was only observed for certain daughter fragments. Without an internal mass signature, it is not straightforward to deduce the peptide sequence from this particular fragment pattern behavior in comparison to the fragment assignment for daughter ions all with lysine at the C-terminus. However, with the intrinsic lys- $d_{4}$ tags, the lysine-containing sister fragments were easily tracked. For example, the $\mathrm{M}^{+}$peak at 1243.7175 has a 4-Da split, indicating one lysine for this fragment, whereas, another $\mathrm{M}^{+}$ion at (a)

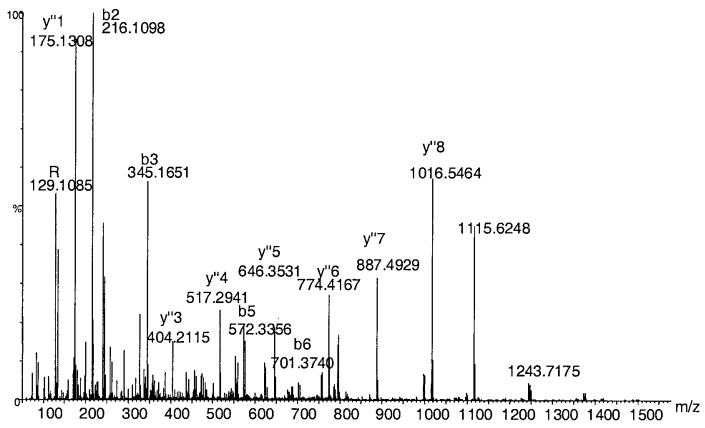

(b)

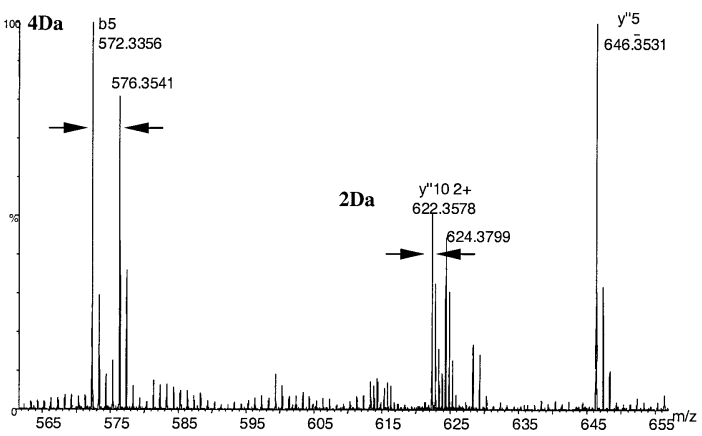

Figure 3. Q-TOF MS/MS spectra of ions with $\mathrm{m} / \mathrm{z} 1587.798$ and its labeled form 1591.823 from the $50 \%$ lys- $d_{4}$ labeled protein mixture. (a) MS/MS spectrum for the range $\mathrm{m} / \mathrm{z} 50$ to 1550 . The sequencing result (TNEKVELQELNDR) was confirmed by protein identification using BioLynx (Micromass). All the fragment ions $\mathrm{b} 4-\mathrm{b} 12$ and $\mathrm{y} 10-\mathrm{y} 12$ containing a lysine residue showed the characteristic mass splits. (b) Expanded $\mathrm{m} / \mathrm{z}$ view of the MS/MS spectrum from $\mathrm{m} / \mathrm{z} 560$ to 660 . The effects of lys- $d_{4}$ tagging were clearly observed (4 Da split indicates singly charged ions, $2 \mathrm{Da}$ split indicates doubly charged ions).

1115.6248 displays no split showing that it lacks lysine. The mass difference between these two adjacent peaks is $128.0927 \mathrm{Da}$, which is approximately equal to the mass of a lysine residue. Thus, the daughter ion at 1115.6248 Da results from the loss of a lysine from the ion at $1243.7175 \mathrm{Da}$. Using lys- $d_{4}$ to label all lysinecontaining daughter ions, the precise peptide sequence was determined as TNEKVELQELNDR with an internal lysine at a missed cleavage site, giving some masssplit signals for both C- and N-terminal fragments. Significantly, these internal lysine(s) which are not cleaved can not be identified by any chemical modification strategies such as MCAT. However, intrinsically incorporated lys- $d_{4}$ labels can resolve these ambiguous peptide sequences de novo. Further, the BioLynx search engine was easily constrained by the lysine identifier which greatly improved both the sequence coverage and the fragment matching scores in protein identification (data not shown).

The specificity of the lys- $d_{4}$ tagging approach can also indirectly increase mass accuracy for the precise discrimination of residues with small mass differences such as lysine and glutamine. For example, a de novo peptide sequence determined from a Q-TOF MS/MS 


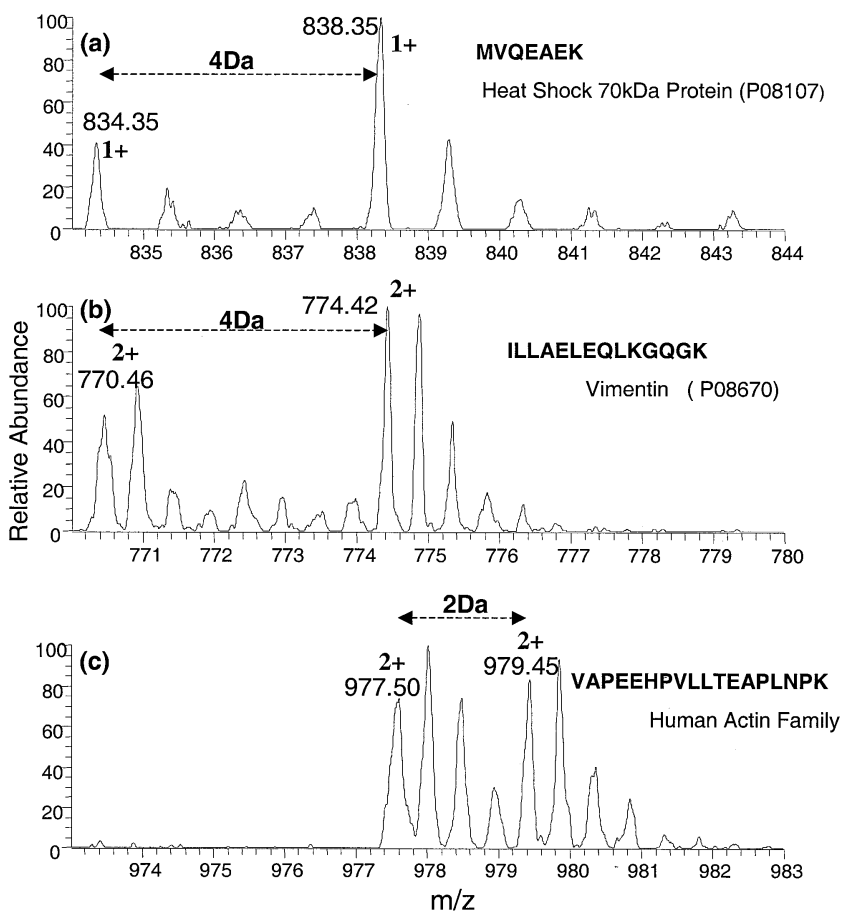

Figure 4. The lys- $d_{4}$-assisted quantitative analysis of proteins from the 1-D gel electrophoresis of the cell lysate from a 1:1 mixture ratio of unlabeled control cells and the $100 \%$ lys- $d_{4}$ labeled heat-treated cells. Each spectrum shows a doublet peak spacing 4 or $2 \mathrm{Da}$. The $\mathrm{m} / \mathrm{z}$ difference between the light and heavy peaks is dependent on the number of lysine residue(s) in the peptide and its charged state; 2.0 Da for a doubly charged peptide containing one lysine, 4.0 Da for a singly charged peptide containing one lysine or a doubly charged peptide with two lysines. (a) Averaged zoom scan spectrum of the peptide with $\mathrm{m} / \mathrm{z}$ at 834.35 from an in-gel digestion of the 1-D SDS PAGE gel band at $70 \mathrm{kDa}$. Lys- $d_{4}$-assisted de novo sequencing identified the peptide as that from the HSP70 protein. The intensity ratio between peak at 834.35 (untreated, non-labeled peptide) and 838.35 (heat treated, lys- $d_{4}$ labeled peptide) is about 1:2.5, indicating the HSP70 protein expression level was induced 2.5-fold by heat treatment. (b) Averaged zoom scan spectrum of the peptide with $\mathrm{m} / \mathrm{z}$ at 770.46 from the in-gel digestion of the 1-D SDS PAGE gel band at $55 \mathrm{kDa}$. The expression level of the identified protein, vimentin, was induced by 2-fold. (c) Averaged zoom scan spectrum of the peptide with $\mathrm{m} / \mathrm{z}$ at 977.50 from in-gel digestion of 1-D SDS PAGE gel band at $40 \mathrm{kDa}$. The expression level of the identified protein, actin, was unaffected by heat treatment.

experiment was VES(L/I)KEE(L/I)AF(L/I)K by BioLynx. However, the intact peptide and its daughter fragments all showed a 4-Da mass-split confirming the presence of only one lysine at the C-terminus. The lys- $d_{4}$ tagging approach gave the correct sequence for this peptide as VES(L/I)QEE(L/I)AF(L/I)K identifying the human protein as vimentin. Thus the high specificity of lys- $d_{4}$ tagging enables it to distinguish protein mutants resulting from genomic polymorphisms or stress, and also to correct the misidentification of proteins resulted from genomic sequence errors or ambiguities.

Protein quantification using lys- $d_{4}$ labels was demonstrated by the human heat shock response. HSF cells grown in $100 \%$ lys- $d_{4}$ labeled medium were heat-treated and mixed with an equal number of control, unlabeled cells grown under normal condition. As shown in Figure 4, the lysine-containing peptides were identified through the characteristic 4-Da mass split and their sequences and parent proteins determined by lys- $d_{4^{-}}$ assisted de novo sequencing. Figure 4a shows an averaged zoom-scan ESI-LCQ spectrum of a lysine-contain- ing peptide of HSP70. Its unlabeled counterpart from control cells is at $834.35 \mathrm{Da}$ and its lys- $d_{4}$-labeled form from the heated-treated cells is at $838.35 \mathrm{Da}$. The relative ratio of the labeled and unlabeled peak intensities indicates that the heat shock induced a 2.5 -fold increased expression of the HSP70 protein. Similarly, the heat shock treatment induced increased expression of vimentin 2-fold as shown by the signal ratio of the peaks at 770.46 and 774.42 (Figure $4 \mathrm{~b}$ ). This incompletely digested peptide is double-charged and with a 4-Da mass-split for the two lysines it contains. As a control, a tryptic peptide from the actin family of proteins gave peaks of equal intensity for both the labeled and unlabeled forms at 977.50 and $979.45 \mathrm{Da}$, respectively (Figure 4c), showing no heat shock response. The major uncertainties include the deuterium purity of the $d_{4}$-labeled lysine precursors, and the accuracy of cell mixing for the control and the heattreated cells, etc. Because of the higher abundance of lysine in cellular proteins in comparison to cysteine residues that is the target for ICAT, usually several 
lysine-containing peptide signals from each protein can be observed in a single LC-MS profile-run. In our design, we have minimized these errors by plotting a standard curve of a series of the lys- $d_{4}$ peptides from each protein for calibration. The precision of protein quantification using our approach was determined at $\pm 5 \%$. These results were well in agreement with previous studies in regards to the response of these proteins to heat shock [14-16].

The use of lys- $d_{4}$ as a label has many unique advantages; first, lysine residues in human proteins are more widely distributed and in higher abundance than cysteinyl residues that are the primary target for the ICAT reagent. Thus, this label gives much larger sequence coverage, allowing protein identifications with enhanced confidence; second, lysine residues are specifically located at the C-termini of many trypsin- or Lys-C proteolytic peptides and, when labeled, their daughter ions can be easily tracked in tandem MS/MS experiments; third, the mass increase of lys- $d_{4}$ allows lysine to be easily distinguished from glutamine, thus resolving this type of sequence ambiguity; fourth, compared to tagging proteins with unnatural chemicals, lys- $d_{4}$ labels represent an intrinsic component of cellular proteins. Labeling with lys- $d_{4}$ during in vivo cell growth is a natural process that avoids problems of specificity or efficiency of labeling and the nonspecific in vitro chemical labeling of other amino acids; therefore, the use of lys- $d_{4}$ allows many basic biological questions to be addressed at the cell and molecular levels.

Both ICAT and MCAT methods have considerably improved the specificity and throughput of peptide sequencing and protein quantitation by chemically tagging cysteine or lysine residues. However, the culturing of cells in defined media is essential in understanding the molecular mechanisms of cellular responses to environmental stresses, pathogenic infections, and the cellular responses to therapeutics using MS-based proteomic approaches. Due to the high sensitivity of MS, the cost of the labeled amino acids is minimum for each small-scale cell culture. Because the deuterium-labeled lysine precursors have the same chemical structure and physical properties as the naturally occurring lysine residues, except for their difference in mass, the incorporation of lys- $d_{4}$ in human cells was both highly efficient and specific. More importantly, the presence of lys- $d_{4}$ in peptides has no affect on their MS/MS fragmentation, signal ionization, and fragment assignment.

In comparison to the method using ${ }^{15} \mathrm{~N}$ isotope as an internal reference for protein quantification [17], the defined value of lys- $d_{4}$ tags can avoid unpredictable mass shifts for proteolytic peptides caused by the uniform ${ }^{15} \mathrm{~N}$ labeling of every amino acid residue in the proteins. The 4-Da mass-shift pattern of lys- $d_{4}$ labels is easily recognizable, which allows accurate quantification by observing the unlabeled and labeled peptide simultaneously in a single zoom scan on LCQ MS. While ICAT-based protein quantification is restricted to the low natural abundance of cysteine-containing peptides, the more abundant lysine content will ensure that all protein signals can be included in the analysis. Superior to the MCAT approach that uses chemically modified lysines as the signal reference for quantitation, both the unlabeled and lys- $d_{4}$-labeled species have the same ionization efficiency that provides a reliable comparison of signal intensities. In conclusion, our strategy of in vivo lys- $d_{4}$ tagging is fully amenable to automation for a large-scale analysis and is particularly useful for the systematic investigation of complex proteomes such as the human proteome.

\section{Acknowledgments}

The authors thank Dr. Anders Lund for assistance with the experiments on Micromass Q-TOF. This work was supported by DOE Human Genome Instrumentation grant ERW9840, Los Alamos National Laboratory LDRD grant 200071, and DOE grant KP1103010 (to XC). XC is a recipient of the Presidential Early Career Award for Scientists and Engineers (PECASE).

\section{References}

1. Gygi, S. P.; Rist, B.; Gerber, S. A.; Turecek, F.; Gelb, M. H.; Aebersold, R. Quantitative Analysis of Complex Protein Mixtures Using Isotope-Coded Affinity Tags. Nat. Biotechnol. 1999, 17, 994-999.

2. Cagney, G.; Emili, A. De Novo Peptide Sequencing and Quantitative Profiling of Complex Protein Mixtures Using MassCoded Abundance Tagging. Nat. Biotechnol. 2002, 20, 163-170.

3. Wilm, M.; Shevchenko, A.; Houthaeve, T.; Breit, S.; Schweigerer, L.; Fotsis, T.; Mann, M. Femtomole Sequencing of Proteins from Polyacrylamide Gels by Nano-Electrospray Mass Spectrometry. Nature 1996, 379, 466-469.

4. Zhang, Z.; McElvain, J. S. De Novo Peptide Sequencing by Two-Dimensional Fragment Correlation Mass Spectrometry. Anal. Chem. 2000, 72, 2337-2350.

5. Shevchenko, A.; Chernushevich, I.; Ens, W.; Standing, K. G.; Thomson, B.; Wilm, M.; Mann, M. Rapid "de Novo" Peptide Sequencing by a Combination of Nano-Electrospray, Isotopic Labeling, and a Quadrupole/Time-of-Flight Mass Spectrometer. Rapid Commun. Mass Spectrom. 1997, 11, 1015-1024.

6. Lindh, I.; Hjelmqvist, L.; Bergman, T.; Sjovall, J.; Griffiths, W. J. De Novo Sequencing of Proteolytic Peptides by a Combination of C-Terminal Derivatization and Nano-Electrospray/ Collision-Induced Dissociation Mass Spectrometry. J. Am. Soc. Mass Spectrom. 2000, 11, 673-686.

7. Goodlett, D. R.; Keller, A.; Watts, J. D.; Newitt, R.; Yi, E. C.; Purvine, S.; Eng, J. K. von; Haller, P.; Aebersold, R.; Kolker, E. Differential Stable Isotope Labeling of Peptides for Quantitation and de Novo Sequence Derivation. Rapid Commun. Mass Spectrom. 2001, 15, 1214-1221.

8. Keough, T.; Youngquist, R. S.; Lacey, M. P. A Method for High-Sensitivity Peptide Sequencing Using Postsource Decay Matrix-Assisted Laser Desorption Ionization Mass Spectrometry. Proc. Natl. Acad. Sci. U.S.A. 1999, 96, 7131-7136.

9. Munchbach, M.; Quadroni, M.; Miotto, G.; James, P. Quantitation and Facilitated de Novo Sequencing of Proteins by Isotopic N-Terminal Labeling of Peptides with a Fragmentation-Directing Moiety. Anal. Chem. 2000, 72, 4047-4057.

10. Keough, T.; Lacey, M. P.; Youngquist, R. S. Derivatization Procedures to Facilitate de Novo Sequencing of Lysine-Terminated Tryptic Peptides Using Postsource Decay Matrix-As- 
sisted Laser Desorption/Ionization Mass Spectrometry. Rapid Commun. Mass Spectrom. 2000, 14, 2348-2356.

11. Qin, J.; Herring, C. J.; Zhang, X. De Novo Peptide Sequencing in an Ion Trap Mass Spectrometer with $18 \mathrm{O}$ Labeling. Rapid Commun. Mass Spectrom. 1998, 12, 209-216.

12. Zhu, H.; Hunter, T. C.; Pan, S.; Yau, P. M.; Bradbury, E. M.; Chen, X. Residue-Specific Mass Signatures for the Efficient Detection of Protein Modifications by Mass Spectrometry. Anal. Chem. 2002, 74, 1687-1694.

13. Hunter, T. C.; Yang, L.; Zhu, H.; Majidi, V.; Bradbury, E. M.; Chen, X. Peptide Mass Mapping Constrained with Stable Isotope-Tagged Peptides for Identification of Protein Mixtures. Anal. Chem. 2001, 73, 4891-4902.

14. Vilaboa, N. E.; Garcia-Bermejo, L.; Perez, C.; de Blas, E.; Calle, C.; Aller, P. Heat-Shock and Cadmium Chloride Increase the
Vimentin mRNA and Protein Levels in U-937 Human Promonocytic Cells. J. Cell Sci. 1997, 110(Part 2), 201-207.

15. Schiaffonati, L.; Tacchini, L.; Pappalardo, C. Heat Shock Response in the Liver: Expression and Regulation of the hsp70 Gene Family and Early Response Genes After in Vivo Hyperthermia. Hepatology 1994, 20, 975-983.

16. Dressel, R.; Johnson, J. P.; Gunther, E. Heterogeneous Patterns of Constitutive and Heat Shock Induced Expression of HLALinked HSP70-1 and HSP70-2 Heat Shock Genes in Human Melanoma Cell Lines. Melanoma Res. 1998, 8, 482-492.

17. Oda, Y.; Huang, K.; Cross, F. R.; Cowburn, D.; Chait, B. T. Accurate Quantitation of Protein Expression and Site-Specific Phosphorylation. Proc. Natl. Acad. Sci. U.S.A. 1999, 96, 65916596. 